\title{
Implementation and applications of a finite-element model for the contact between rough surfaces
}

\section{Poulios, Konstantinos; Klit, Peder}

Published in:

Wear

Link to article, DOI:

10.1016/j.wear.2013.02.024

Publication date:

2013

Link back to DTU Orbit

Citation (APA):

Poulios, K., \& Klit, P. (2013). Implementation and applications of a finite-element model for the contact between rough surfaces. Wear, 303(1-2), 1-8. https://doi.org/10.1016/j.wear.2013.02.024

\section{General rights}

Copyright and moral rights for the publications made accessible in the public portal are retained by the authors and/or other copyright owners and it is a condition of accessing publications that users recognise and abide by the legal requirements associated with these rights.

- Users may download and print one copy of any publication from the public portal for the purpose of private study or research.

- You may not further distribute the material or use it for any profit-making activity or commercial gain

- You may freely distribute the URL identifying the publication in the public portal

If you believe that this document breaches copyright please contact us providing details, and we will remove access to the work immediately and investigate your claim. 


\title{
Implementation and applications of a finite-element model for the contact between rough surfaces
}

\author{
K. Poulios ${ }^{\mathrm{a}, *}$, P. Klit ${ }^{\mathrm{a}}$ \\ ${ }^{a}$ Technical University of Denmark, Department of Mechanical Engineering, Solid Mechanics Nils \\ Koppels All, Building 404, 2800 Kgs. Lyngby
}

\begin{abstract}
Due to the rough nature of real mechanical surfaces, the contact between elastic bodies occurs at several size scales. Statistical and fractal contact models can take a wide range of roughness wavelengths into account, without additional computational cost. However, deterministic models are more straightforward to understand and easier to extend to more complex cases like contacting bodies that demonstrate elasto-plastic behaviour. This paper presents a finite-element model for studying the frictionless contact between nominally flat rough surfaces. Apart from a description of the model implementation, results from a series of calculations corresponding to theoretical and real life applications are included. Numerically generated surface topographies but also roughness measurements from a stylus instrument are used as input for the model. Elastic and perfectly elastic-plastic materials are included in the examples. Among the presented results one can find the distribution of the contact pressure at the interface and diagrams of the real area of contact as a function of the nominal contact pressure.
\end{abstract}

Keywords: real contact area, finite-element, micro-contact

\section{Introduction}

Problems in contact mechanics can be roughly classified into two categories. The ones that refer to non-conformal contacting surfaces and those that treat the contact between macroscopically conformal surfaces. The latter case can often be reduced to a contact between rough but nominally flat surfaces. This kind of problem has many real life applications involving friction, wear and electrical or thermal conduction and was extensively studied since the classical works of Bowden \& Tabor [1], Archard [2] and Greenwood \& Williamson [3].

The real area of contact was very early identified as a key parameter in understanding and quantifying the phenomena that occur in the contact between conformal rough surfaces. For this reason a big number of either statistical or deterministic models were suggested for the calculation of the real area of contact and possibly the corresponding

\footnotetext{
*Corresponding author

Email address: kopo@mek.dtu.dk (K. Poulios)
} 
pressure distribution across and within a large number of contact spots. Statistical and fractal models work with surface parameters while deterministic models require a representative surface sample in form of a topography matrix that is either measured or numerically generated.

Most of the fundamental questions that modern models for contact between rough surfaces are expected to solve in a quantitative manner, are already discussed in the three classical papers cited above. The role of the real contact area in the calculation of an approximately load independent coefficient of friction is highlighted in [1. Moreover, even if that work overestimated the importance of plastic deformation in the asperities contact, plastic deformation is still a parameter which modern models often need to take into account in order to represent reality precisely. Archard in [2] states the importance of the surface topography in predicting a nearby linear relation between the normal load and the real contact area even under purely elastic deformations. For approximating such a relation he resorts to a multi-scale consideration of the surface roughness, in a way similar to modern fractal models. Finally Greenwood \& Williamson show in 3 that an approximately linear relation between the real contact area and the normal load can be demonstrated even in a single size-scale consideration if a realistic statistical distribution of the asperities heights is taken into account. Ever since, this is recognized as the major effect in predicting how the real area of contact grows with the load.

More recent rough surface contact models consist to a large extent in refinements of the Greenwood \& Williamson model, mainly in the directions of including inter-asperity interactions, variable asperity curvature and plasticity, see e.g. [4] and [5]. Models that intend to include multiple size-scales normally deviate from the Greenwood \& Williamson approach. Typical examples are the fractal model proposed by Majumdar \& Bhushan in [6] and the one introduced by Persson in 7.

With respect to deterministic contact models, most of them are based on either an elastic half-space or a finite-element formulation, see e.g. [8, 9] and [10, 11] respectively. The present work includes implementation details and applications of a finite-element based model, that is capable of calculating the deformations, the contact pressure distribution and the real contact area between flat rough surfaces of two elasto-plastic bodies that are in contact under normal loading.

\section{Methods}

\subsection{Continuous problem formulation}

In continuum mechanics, the deformations $u_{1}$ and $u_{2}$ of two elastic bodies identified through the indices $i=1$ and 2 can be studied by considering the following equations:

$$
\begin{aligned}
& \nabla \sigma_{i}\left(u_{i}\right)+f_{i}=0 \\
& \sigma_{i}\left(u_{i}\right)=\mathcal{A}_{i} \epsilon_{i}\left(u_{i}\right)
\end{aligned}
$$

in the corresponding domains $\Omega_{i}$ shown e.g. in Fig. 1

With $\sigma$ denoting the three by three stress tensor, Eq. (1) expresses the forces equilibrium in the bulk material under field forces $f$ and Eq. (2) represents a constitutive law for elastic deformations with $\mathcal{A}$ and $\epsilon$ corresponding to the elasticity and the linearized strain tensors respectively. 


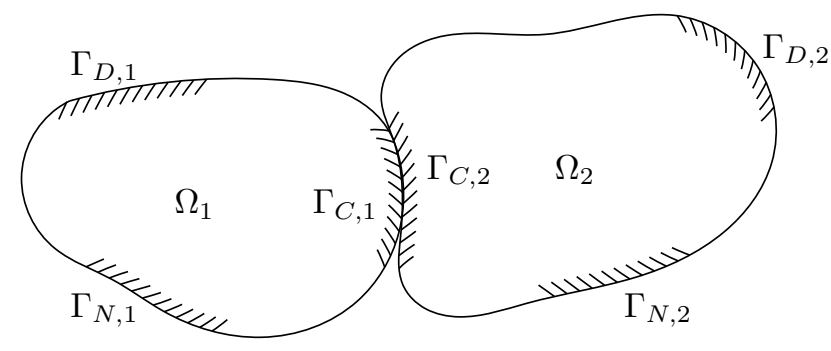

Figure 1: Deformable bodies in contact

Additionally, homogeneous Dirichlet conditions on the boundaries $\Gamma_{D, i}$ and surface normal forces $t_{i}$ on the boundaries $\Gamma_{N, i}$ can be expressed through the following equations respectively:

$$
\begin{gathered}
u_{i}=0, \text { on } \Gamma_{D, i} \\
\sigma_{N, i}=t_{i}, \text { on } \Gamma_{N, i}
\end{gathered}
$$

with the stress component $\sigma_{N, i}$ in direction $n_{i}$ normal to the body surface, defined as:

$$
\sigma_{N, i}\left(u_{i}\right)=\sigma_{i}\left(u_{i}\right) n_{i}
$$

For studying the contact interaction between the two elastic bodies, the non-penetration condition can be described on a portion $\Gamma_{C, 1}$ of the surface of the first body by using the so-called Karush-Kuhn-Tucker conditions:

$$
\begin{gathered}
\sigma_{N, 1}\left(u_{1}\right) \leq 0, \text { on } \Gamma_{C, 1} \\
g_{N}\left(u_{1}, u_{2}\right) \geq 0, \text { on } \Gamma_{C, 1} \\
g_{N}\left(u_{1}, u_{2}\right) \sigma_{N, 1}\left(u_{1}\right)=0, \text { on } \Gamma_{C, 1}
\end{gathered}
$$

with the normal gap $g_{N}$ between the bodies, defined as:

$$
g_{N}\left(u_{1}, u_{2}\right)=g_{N 0}-\left(u_{1}-P_{1}\left(u_{2}\right)\right) n_{1}
$$

The variable $g_{N 0}$ corresponds to the initial gap between the unloaded bodies and $P_{1}$ represents a projection operation on the surface of the first body. In the case of Eq. (9) the projected quantity is the displacement field $u_{2}$.

Eq. (6) enforces that only compressive stresses can be exerted between the contacting bodies and Eq. (7) enforces the actual non-penetration condition. Finally, Eq. (8) expresses that at a specific point either contact will occur or the contact pressure will be zero. A more detailed description of the fundamental problem formulation can be found in 12 . 


\subsection{Finite-element discretization}

For geometrically complex contacting bodies, the problem stated in the previous section cannot be solved analytically and is typically discretized by using the finiteelement method. In the case of a nominally flat rough surface and for relatively small roughness asperity slopes the complexity of the geometry cannot justify resorting to a finite-element model, half-space models can solve this problem in a more efficient way, see e.g. 9]. However, if the effect of asperity slopes has to be taken into account or if the bulk material is expected to deform plastically, the finite-element method provides a very straightforward approach in solving this problem.

Two further steps that are typically required before the actual discretization are the conversion of the problem statement into the corresponding weak formulation and the introduction of Lagrange multipliers in Eq. (6) to (8). Both these steps are described in detail in [13] and [14]. With respect to the discretization of the contact conditions, the latter paper describes two possible approaches, a nodal one that enforces Eq. (6) to (8) at each finite-element node and an integral one that enforces the condition in an average sense over the contacting face of each finite-element. In the present work the integral approach is used.

The final form of this problem after its conversion into a series of linear steps according to the Generalized-Newton-Method algorithm presented in [13], is described below:

$$
\begin{gathered}
{\left[\begin{array}{ccc}
K_{1}^{k} & 0 & C_{N, 1} \\
0 & K_{2}^{k} & C_{N, 2} \\
B_{N, 1}^{k} & B_{N, 2}^{k} & D^{k}
\end{array}\right] \cdot\left[\begin{array}{l}
\delta u_{1} \\
\delta u_{2} \\
\delta \lambda_{N}
\end{array}\right]=\left[\begin{array}{l}
R_{1}^{k} \\
R_{2}^{k} \\
R_{\lambda}^{k}
\end{array}\right]} \\
{\left[\begin{array}{c}
u_{1}^{k+1} \\
u_{2}^{k+1} \\
\lambda_{N}^{k+1}
\end{array}\right]=\left[\begin{array}{c}
u_{1}^{k} \\
u_{2}^{k} \\
\lambda_{N}^{k}
\end{array}\right]+\alpha \cdot\left[\begin{array}{l}
\delta u_{1} \\
\delta u_{2} \\
\delta \lambda_{N}
\end{array}\right]}
\end{gathered}
$$

with the index $k$ corresponding to the current step of the iterative solving process.

The factor $\alpha$ for calculating the next iteration step is determined by performing a line search operation. For linear elastic bulk materials the stiffness sub-matrices $K_{i}$ are actually independent of the iteration index $k$. However, for elasto-plastic materials they have to be recalculated at every iteration and can be expressed as $K_{i}\left(u_{i}\right)$. Submatrices $C_{N, i}$ are constant and sub-matrices $B_{N, i}$ and $D$ change as the contact area changes during the numerical iterations and can actually be written as $B_{N, i}\left(u_{1}, u_{2}, \lambda_{N}\right)$ and $D\left(u_{1}, u_{2}, \lambda_{N}\right)$. The right-hand-side terms of Eq. (10) are also functions of $u_{1}, u_{2}$ and $\lambda_{N}$.

All components that were described in this subsection are available in the public domain finite-element library GetFem ++1 that was utilized for the implementation of the present model.

\subsection{Mesh generation}

Fig. 2 shows the mesh discretization used in the model consisting of two rectangular parallelepiped blocks corresponding to the two contacting bodies. Each block is discretized using 8-node hexahedral elements and is divided in layers of different mesh size.

$\sqrt[1]{\text { http://download.gna.org/getfem/html/homepage/ }}$ 


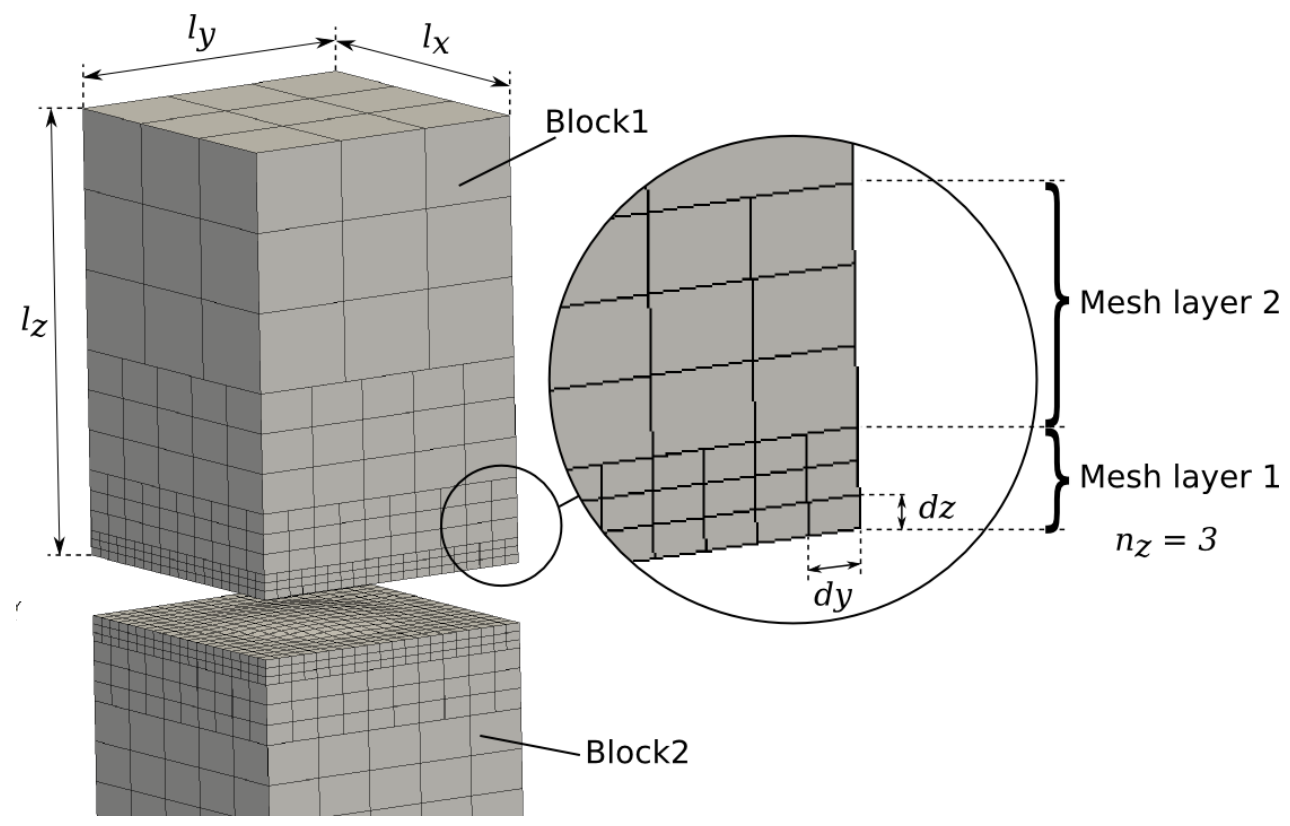

Figure 2: Finite-element mesh

Instead of using transition elements, the continuity between the non-matching meshes of neighbouring layers is enforced by applying additional constraints using Lagrange multipliers defined on the finite-element nodes of the finest of the neighbouring layers. More details and examples about this technique for connecting non-matching meshes can be found in 15 .

The parameters describing the mesh of each block consist of the block dimensions $l_{x}$, $l_{y}, l_{z}$, the number of elements in z direction per layer, $n_{z}$, and the mesh sizes $d x, d y$ and $d z$ at the finest mesh layer. The mesh size in the other layers is determined by a coarsening factor as close to the value of two as possible and the number of layers is such that the prescribed block height $l_{z}$ is fulfilled.

For each block, the roughness topography of the contacting surface is read as a matrix stored in a file and is applied to the nodes of the finest mesh layer as displacements in the $\mathrm{z}$ direction weighted by a factor of one for the nodes on the surface and decreasing to zero for the nodes at the interface with the second mesh layer.

\subsection{Boundary conditions}

All results presented later in this paper correspond to a normal loading between the upper and the lower blocks. This condition is achieved by fixing the $\mathrm{z}$ displacement of all nodes on the bottom surface of the lower block and loading the top surface of the upper block with a uniform load in $\mathrm{z}$ direction. The movement in $\mathrm{x}$ and $\mathrm{y}$ directions for both the bottom surface of the lower block and the top surface of the upper block is restricted in an average sense, this means that the individual nodes are free to move in these directions but the average movement over each surface is zero. 
The blocks shown in Fig. 2 correspond to only a small, possibly representative, portion of the two bodies in contact. In order to take this into account and assuming that macroscopically the contact pressure distribution is uniform, periodicity conditions are introduced to the model. The lateral block surfaces in the positive and negative $\mathrm{x}$ directions are connected together and this is also the case for the other two lateral surfaces perpendicular to the $\mathrm{y}$ direction. The connection is implemented through elimination of finite-element nodes. The DOFs corresponding to one of the connected surfaces are removed and replaced by the corresponding DOFs of the opposite surface. The resulting model should ultimately be interpreted as a rectangular array of infinite blocks like the ones shown in Fig. 2 extending in $\mathrm{x}$ and $\mathrm{y}$ directions.

\subsection{Material properties}

Two different material laws, that were already implemented in the GetFem ++ library, are supported by the present model. Ideally elastic isotropic materials, expressed by Eq. (2), and perfectly elastic-plastic materials can be taken into account.

Plastic deformation can be considered only in the context of a time dependent process, where the previous time or load step provides the current deformations and stresses, $u^{0}$ and $\sigma^{0}$ respectively, as a reference. In this case, a hypothetical elastic relationship between the deformations and stresses can be expressed in incremental sense with respect to the current state as:

$$
\tilde{\sigma}(u)=\sigma^{0}+\mathcal{A}\left(\epsilon(u)-\epsilon\left(u^{0}\right)\right)
$$

Eq. (12) is equivalent to Eq. (2) with the body index $i$ omitted for the sake of simplicity. According to the closest-point projection method described in [16, chap. 10.7], plastic behavior of a material can be taken into account by projecting the hypothetical elastic stresses $\tilde{\sigma}$ to the space of admissible stresses. By introducing the yield limit $\sigma_{y}$ with respect to the Von Mises yield criterion, the constitutive law for a perfectly elastic-plastic material behavior can be expressed based on Eq. [12, as:

$$
\sigma(u)=\left\{\begin{array}{cl}
\tilde{\sigma} & , \text { for } \tilde{\sigma}_{\mathrm{vm}} \leq \sigma_{y} \\
\tilde{\sigma}_{m}+\frac{\sigma_{y}}{\tilde{\sigma}_{\mathrm{vm}}} \cdot \tilde{\sigma}_{d} & , \text { for } \tilde{\sigma}_{\mathrm{vm}}>\sigma_{y}
\end{array}\right.
$$

whereby, the hydrostatic and deviatoric stress tensors, $\tilde{\sigma}_{m}$ and $\tilde{\sigma}_{d}$, are defined respectively as following:

$$
\begin{gathered}
\tilde{\sigma}_{m}=\frac{\tilde{\sigma}_{11}+\tilde{\sigma}_{22}+\tilde{\sigma}_{33}}{3} \cdot I \\
\tilde{\sigma}_{d}=\tilde{\sigma}-\tilde{\sigma}_{m}
\end{gathered}
$$

and the scalar function representing the Von Mises stresses can be defined using the euclidean norm of $\tilde{\sigma}_{d}$ :

$$
\tilde{\sigma}_{\mathrm{vm}}=\sqrt{3 / 2} \cdot\left|\tilde{\sigma}_{d}\right|
$$

Considering the incremental nature of plasticity phenomena, if at least one of the contacting bodies is expected to deform plastically, the corresponding Eq. (2) is replaced 
by Eq. 13 and a series of quasi-static load steps, beginning from the unloaded state, has to be simulated.

It should be noted that both the elastic and the elasto-plastic material laws, that were included in the present model, are based on the assumption of geometric linearity that is valid only for small deformations.

\subsection{Performance}

As described in section 2.4 each of the blocks shown in Fig. 2 can be interpreted as part of a periodic space that consists of infinite repetitions of the considered block in form of a rectangular array. In order for the model to represent reality adequately, the dimensions $l_{x}$ and $l_{y}$ of each block must be higher than the longest wavelength of interest that is pronounced on the corresponding real surface. In case that this wavelength can be identified as a deterministic pattern on the surface, the corresponding block dimension has to be an exact multiple of it. Additionally, the mesh discretization sizes $d x$ and $d y$ should be lower than the shortest wavelength of interest on the corresponding real surfaces and directions. For this reason, a very high number of elements $n_{x}=l_{x} / d x$ and $n_{y}=l_{y} / d y$ is necessary in order to cover a range of roughness wavelengths as wide as possible. Therefore, the model size in terms of DOFs and the corresponding computational cost are considerably high.

The following measures were taken in order to reduce the computational cost:

- Use variable mesh size in the depth direction in order to reduce the complexity of the problem.

- Reduce the number of Newton-Raphson iterations by using robust contact algorithms like the one mentioned in section 2.2 .

Additionally, the computational time was reduced by resorting to parallel computing:

- Carry out the assembly of the tangent matrix and the right hand side of Eq. 100 on multiple cpu-cores in parallel.

- Use multiple cpu-cores for the solution of the linear system (10) in each iteration step by utilizing a parallel solver like MUMPS [17] and [18] in our case.

\section{Results and discussion}

In this section a series of examples are presented in order to compare the implemented model with the Greenwood \& Williamson theory, show its utility for real life applications and give an impression about the computational performance that can be achieved utilizing publicly available numerical tools.

\subsection{Comparison with Greenwood \& Williamson}

In order to compare with the Greenwood \& Williamson theory [3], surface topographies that fulfil the corresponding assumptions were generated numerically. Fig. 3 illustrates the sum topography of the contacting surfaces for the four examples presented in this section and Fig. 4 shows the individual topographies corresponding to the upper and lower contacting surfaces for examples 3 and 4 . In all cases the dimensions of the surface 
samples are $l_{x}=1.8$ by $l_{y}=1.8 \mathrm{~mm}$, and the surfaces include 81 spherical bumps of constant radius.

In the first two examples, one of the contacting surfaces is rigid and flat, whereas the other contacting surface is provided with bumps of radius equal to $1 \mathrm{~mm}$ arranged in a rectangular array. The discretization length is $d x=d y=0.02 \mathrm{~mm}$ for the first example and $d x=d y=0.01 \mathrm{~mm}$ for the second one. Apart from the different mesh size, examples 1 and 2 are identical. The heights of the spherical bumps were generated randomly according to a normal distribution with standard deviation of $0.7 \mu \mathrm{m}$. The bulk material is considered isotropic and ideally elastic corresponding to steel against steel and with the Young's modulus $E=1.05 \cdot 10^{5} \mathrm{~N} / \mathrm{mm}^{2}$ and Poisson's ratio $\nu=0.3$ representing an equivalent elastic body in contact with a rigid body.

The third example takes two elastic bodies into account with $E=2.1 \cdot 10^{5} \mathrm{~N} / \mathrm{mm}^{2}$ and $\nu=0.3$, provided with spherical bumps like in the first two examples but with radius equal to $2 \mathrm{~mm}$ and height standard deviations of $0.43 \mu \mathrm{m}$ and $0.55 \mu \mathrm{m}$ for the upper and the lower contacting surfaces respectively. In this way the equivalent radius for the contact between two bumps is equal to $1 \mathrm{~mm}$ and the standard deviation of the sum of the two surfaces will correspond to $\sqrt{0.43^{2}+0.55^{2}} \approx 0.7 \mu \mathrm{m}$. This choice makes this example statistically equivalent to the first two ones.

The fourth example has similar characteristics to the third one. The same material parameters, bump radius and bump height distribution samples were utilized, however the locations of the surface bumps between the two surfaces do not match each other. They are generated by a random function following the uniform distribution with the additional constraint that the overlap with any pre-existing bump will not exceed $40 \%$ of the bump meniscus radius.

The simulation results for examples 1 to 4 , corresponding to applied nominal pressures of $3,6,12,18, \ldots$ up to $48 \mathrm{MPa}$, are summarized in Fig. 5 . In all cases the calculated real area of contact increases almost proportionally with the applied nominal pressure. For a perfectly linear relationship the mean pressure, defined as the total normal load divided by the real contact area, would remain constant. However, the right graph in Fig. 5 shows that there is a small increasing tendency in the mean pressure for increasing nominal pressure.

A comparison of the real contact area and mean pressure curves between examples 1 and 2 shows that the relatively coarse mesh used in the first example can give results that do not deviate more than $5 \%$ from the ones with a twice as fine mesh size. This justifies the use of the same discretization length also in examples 3 and 4 and gives an impression about the expected accuracy for these calculations as well.

The diagrams in Fig. 5 also include the real contact area and the mean pressure calculated according to the Greenwood \& Williamson theory. The match with the numerical results, especially for the fine mesh of example number 2 , is relatively good. The real contact area calculated by the finite-element model seem to be in general lower than the one predicted by the theoretical model. It should be noted however, that the limitation of the Greenwood \& Williamson theory of not accounting for the interaction between the roughness asperities is, by the nature of the finite-element model, not possible to reproduce. Nevertheless, at least for examples 1 to 3, the provided distance between neighbouring bumps indicates that for moderate loads the interaction between them is limited. 


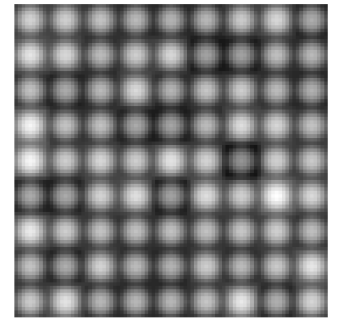

(a) cases 1 and 2

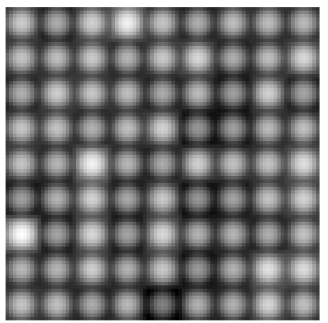

(b) sum for case 3

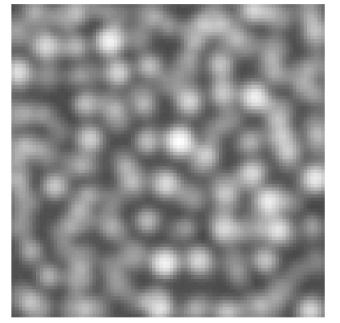

(c) sum for case 4

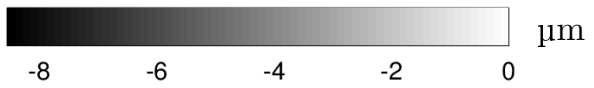

Figure 3: Combined surface topographies used in examples 1-4

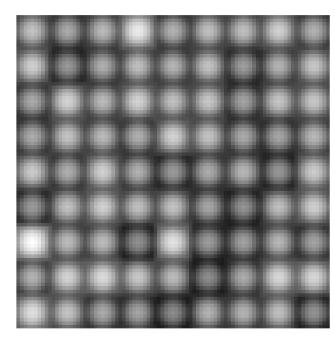

(a) case 3, upper

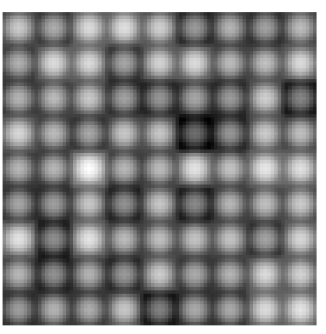

(b) case 3, lower

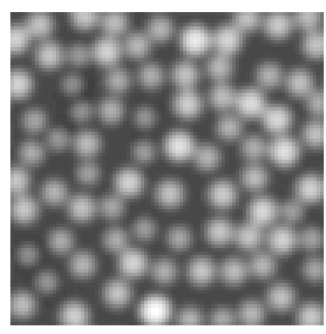

(c) case 4, upper

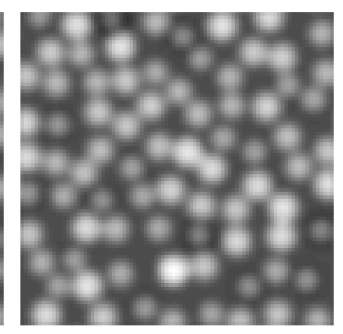

(d) case 4, lower

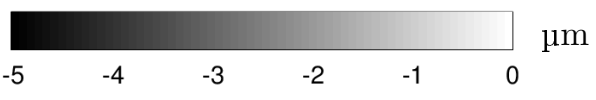

Figure 4: Individual surface topographies used in examples 3 and 4

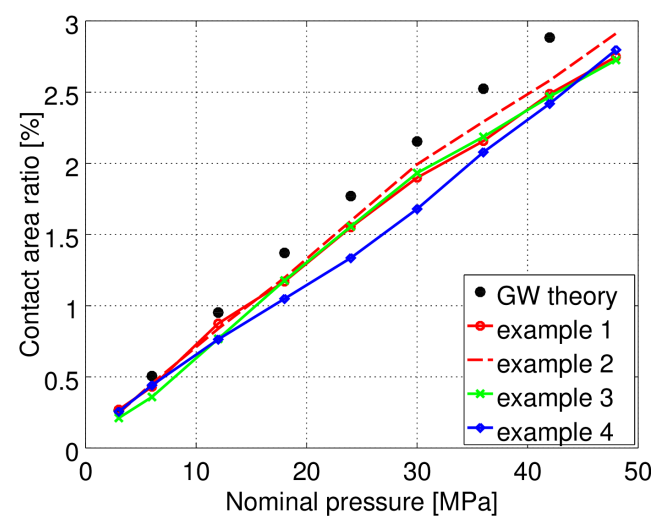

(a)

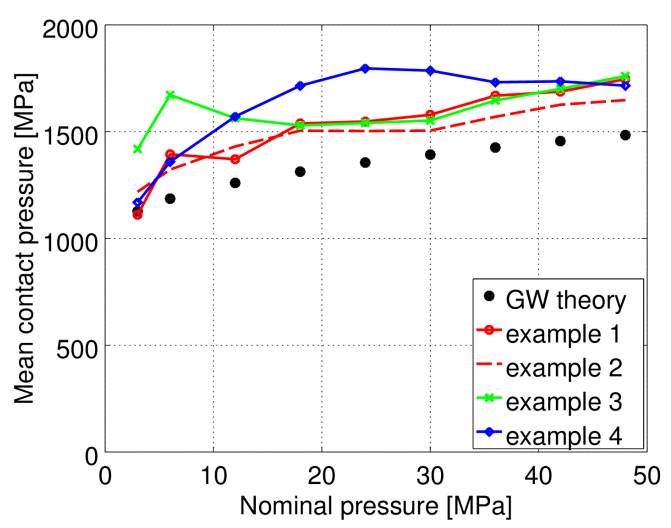

(b)

Figure 5: Calculated real contact area (a) and mean contact pressure (b) for examples 1 to 4 


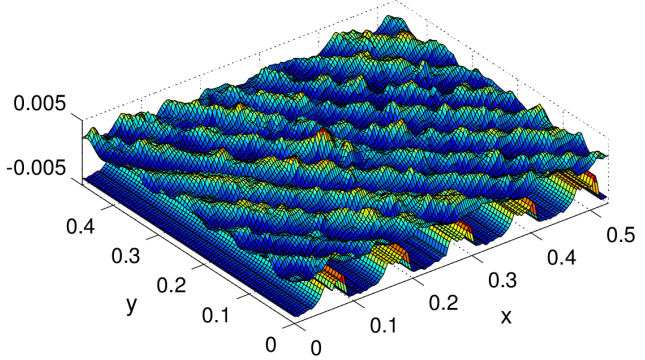

(a) cases 5 and 7

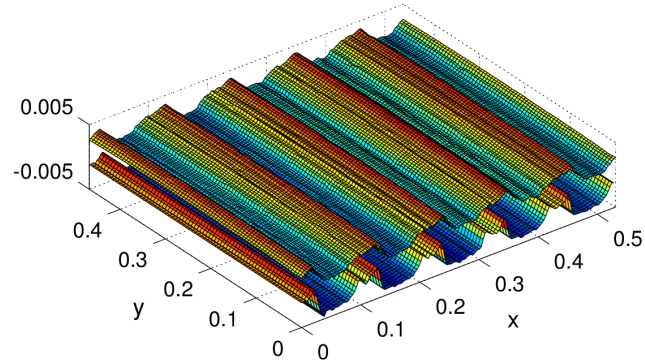

(b) cases 6 and 8

Figure 6: Surface topographies used in examples 5 to 8

\subsection{Polymer against steel}

Examples 5 to 8 refer to surface topographies that were measured on real specimens and are illustrated in Fig. 6. The upper topography on each graph corresponds to the surface of a polyethylene-terephthalate polymer pin used in a pin-on-disc test-rig [19, whereas the lower one represents the corresponding steel disc surface. The left graph illustrates the initial contacting surfaces and the right one shows the measured contacting surfaces after $40 \mathrm{~m}$ of sliding under nominal pressure of $6 \mathrm{MPa}$. In all cases, the dimensions of the surface samples are $l_{x}=0.53$ by $l_{y}=0.47 \mathrm{~mm}$ and the direction of sliding between the pin and the disc is parallel to the y axis. On the initial pin surface turning marks from the machining can be distinguished whereas the final surface seems to have adopted a topography that matches the steel disc surface.

Examples 5 and 6 corresponding to the initial and final surface topographies respectively, were calculated including the plastic deformation of the pin material. The properties of the purely elastic disc material were $E=2.1 \cdot 10^{5} \mathrm{~N} / \mathrm{mm}^{2}$ and $\nu=0.3$, whereas the pin bulk material was assumed to exhibit a perfectly elastic-plastic behaviour with $E=3.5 \cdot 10^{3} \mathrm{~N} / \mathrm{mm}^{2}, \nu=0.43$ and yield strength limit $\sigma_{y}=75 \mathrm{~N} / \mathrm{mm}^{2}$.

Examples 7 and 8 represent the same conditions like cases 5 and 6 respectively with the only modification of removing the yield strength limit and considering the polymer perfectly elastic. This is an academic case serving as a reference for demonstrating the effect of plastic deformation on the real contact area and the solving performance of the model.

The loading conditions for examples 5 and 6 include an increase of the normal load in steps corresponding to nominal pressures of 3,6,12,18 and $24 \mathrm{MPa}$ and a consequent decrease following the same steps in reverse order. Examples 7 and 8 include exclusively elastic materials, hence the decreasing load phase was omitted as it was expected to give identical results as the increasing load phase. Fig. 7 shows the relationship between the applied nominal pressure and the calculated real area of contact on the upper diagrams and the mean contact pressure on the lower diagrams. The diagrams on the left and the right side of the figure refer to the initial and final surface topographies respectively.

One can observe that during the unloading phase of examples 5 and 6 the real area of contact is higher than during loading and consequently the mean contact pressure is lower. The difference between the loading and the unloading branch of the curves 


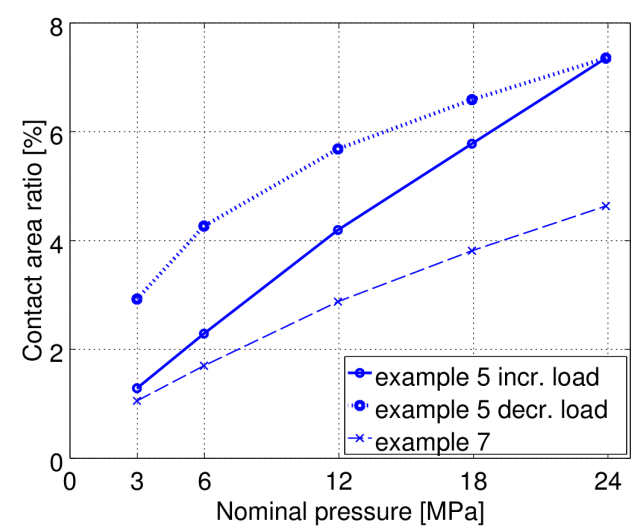

(a)

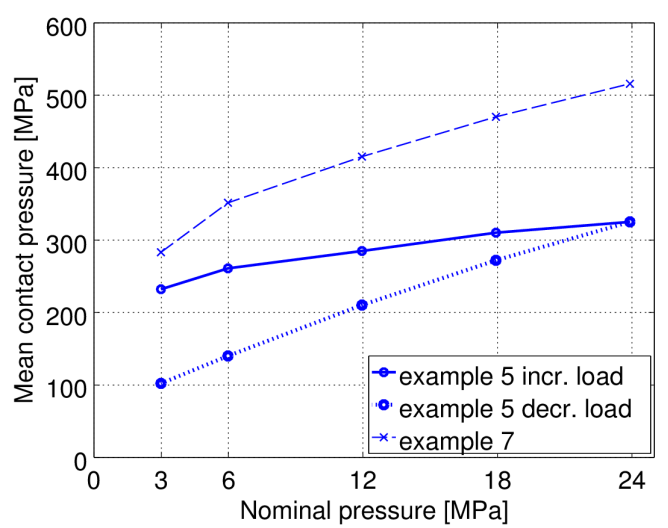

(c)

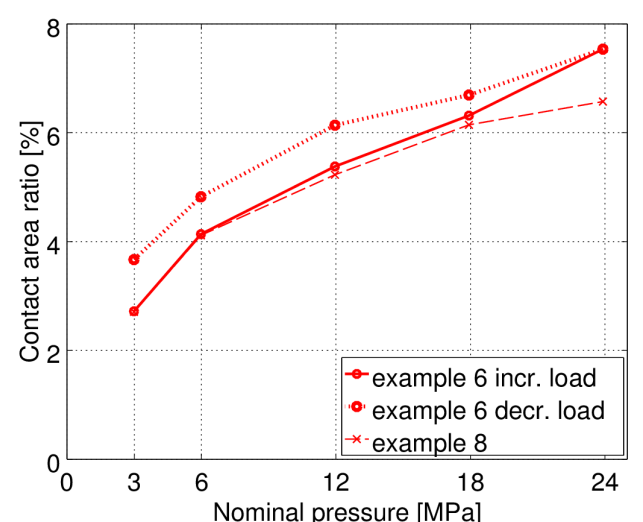

(b)

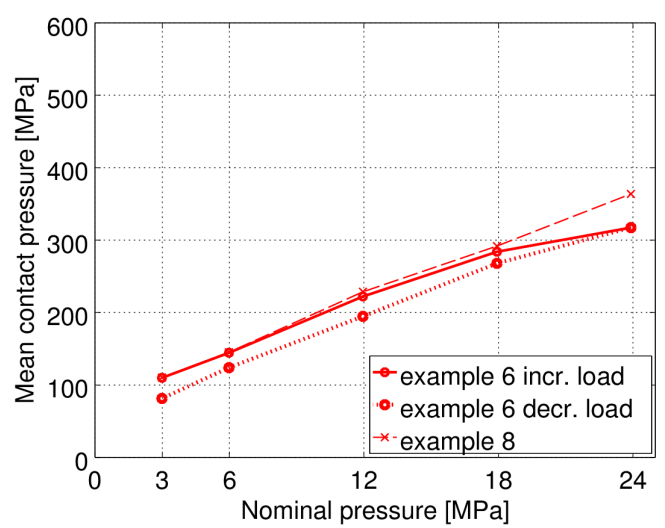

(d)

Figure 7: Calculated real contact area (a,b) and mean contact pressure $(c, d)$ for examples 5 to 8 


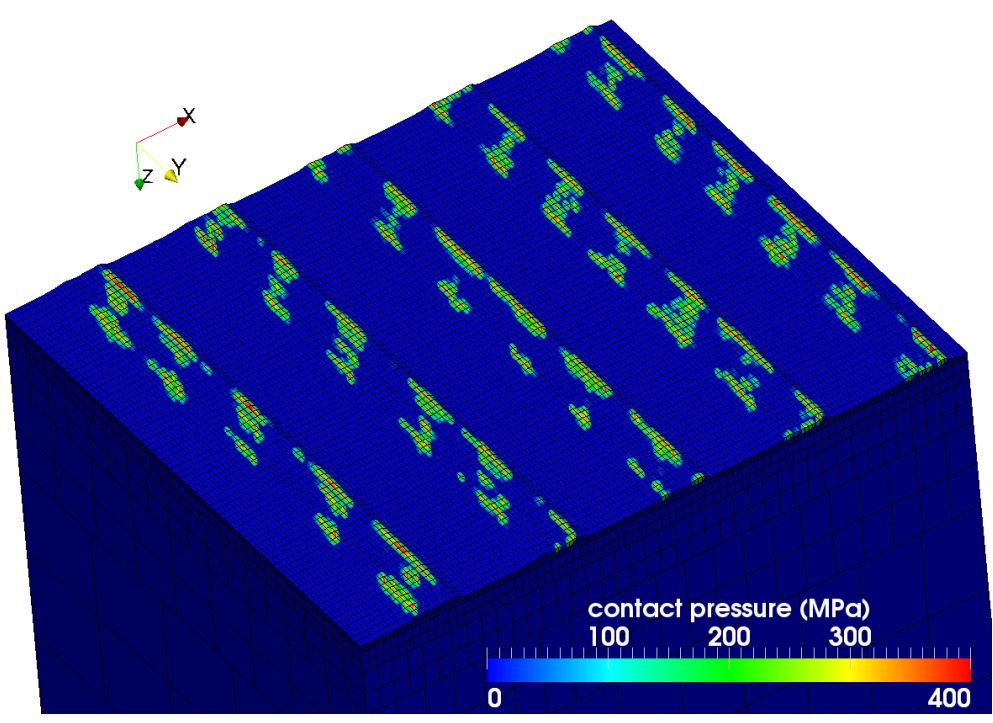

Figure 8: Contact pressure distribution on the disc surface for example 5 at $24 \mathrm{MPa}$

indicates the extend of permanent deformations due to plasticity and it is higher in the case of the initial pin surface (see example 5) than for the final one (see example 6). According to 20, that studies the impact of plastic deformation on the contact area ratio during the unloading phase, the span between the loading and the unloading branch increases with increasing plasticity index $\psi$. It is difficult to define a plasticity index for examples 5 and 6 because the corresponding surfaces do not fulfil the assumptions of 3. However the transition from the surfaces in Fig. 6 (a) to the more conformal ones in Fig. 6 (b) corresponds to a reduced peak curvature radius and consequently to a lower plasticity index.

Examples 7 and 8 show that by neglecting the plastic deformation of the polymer the calculated real area of contact is reduced and the mean contact pressure becomes higher. The difference with respect to examples 5 and 6 respectively is another indicator of the extent of plastic deformation and it is clearly higher in the case of the initial pin surface (compare examples 5 and 7 ).

An interesting observation can be extracted from graph (b) of Fig. 7 which shows that during the loading phase no plastic deformation occurs up to a nominal pressure level of $6 \mathrm{MPa}$. The two curves that were calculated with elastic and elasto-plastic conditions respectively coincide up to this pressure. This is in accordance with the testing conditions of the pin in the real set-up. Since the pin was loaded with a nominal pressure of $6 \mathrm{MPa}$, any plastic deformation that would occur under this load has already occurred during the running-in of the pin within the sliding distance of $40 \mathrm{~m}$. The pin topography shown in Fig 6 (b) should already include this permanent deformation, so that no further plastic deformation is expected without a further increase of the load.

Fig. 8 shows the distribution of the contact pressure over the disc surface for the contact with the original pin under the maximum nominal pressure of $24 \mathrm{MPa}$. The corresponding mean contact pressure that can be read from Fig. 7 (c) is around $325 \mathrm{MPa}$. 


\begin{tabular}{ccccc} 
example & DOFs & $\begin{array}{c}\text { calculation } \\
\text { wall-clock } \\
\text { time }\end{array}$ & $\begin{array}{c}\text { iterations } \\
\text { per load } \\
\text { step }\end{array}$ & $\begin{array}{c}\text { peak } \\
\text { memory } \\
\text { usage }\end{array}$ \\
\hline 1 & 138096 & 1h $24 \mathrm{~min}$ & 3.8 & $0.85 \mathrm{~GB}$ \\
\hline 2 & 551376 & $29 \mathrm{~h} 42 \mathrm{~min}$ & 6.3 & $3.67 \mathrm{~GB}$ \\
\hline 3 & \multirow{2}{*}{267936} & 2h $25 \mathrm{~min}$ & 3.2 & $2.58 \mathrm{~GB}$ \\
4 & & 2h $40 \mathrm{~min}$ & 3.2 & $2.60 \mathrm{~GB}$ \\
\hline 5 & & $12 \mathrm{~h} 47 \mathrm{~min}$ & 10.9 & $4.52 \mathrm{~GB}$ \\
6 & \multirow{2}{*}{426976} & 11h $17 \mathrm{~min}$ & 7.4 & $3.80 \mathrm{~GB}$ \\
7 & & $5 \mathrm{~h} 20 \mathrm{~min}$ & 3.1 & $3.75 \mathrm{~GB}$ \\
8 & & $4 \mathrm{~h} 28 \mathrm{~min}$ & 2.8 & $3.67 \mathrm{~GB}$ \\
\hline
\end{tabular}

Table 1: Summary of model size, calculation time, Newton-Raphson iterations and memory usage

\subsection{Performance}

Table 1 summarizes the sizes of the calculated examples in terms of DOFs, the calculation times, the average number of Newton-Raphson iterations per load-step and the peak memory usage for each calculation. All calculation times in the table are wallclock times for parallel runs on $10 \mathrm{cpu}$-cores. These results should not be considered as accurate benchmarking data but they can serve as indicative values for the overall performance of the model as well as for a rough comparison between the different cases presented above.

Concerning the number of DOFs listed in the table, it should be noted that the major part of the model DOFs describes the displacements field in the contacting blocks. A smaller portion corresponds to Lagrange multipliers that are necessary for describing the contact condition, the continuity between the different mesh layers and the enforcement of the Dirichlet boundary condition at the bottom of the lower block.

A comparison between the first two examples shows that a mesh refinement by a factor of two causes an increase in the number of DOFs by a factor of approximately four. Because of the variable mesh size in the depth direction the total number of DOFs depends on the second instead of the third power of the refinement factor. Nevertheless, the impact of the increased number of DOFs on the computational time is high.

For examples 7 and 8 to be comparable with examples 5 and 6 in terms of performance, the data contained in Table 1 include the decreasing load phase also for these purely elastic cases even if the results are identical with the same load steps during the increasing load phase. It seems that including plasticity for one of the two contacting bodies increases the computation time by a factor around 2.5. This change should be attributed to the similar increase in the number of Newton-Raphson iteration per load step and not to the higher computational cost of the stiffness matrix assembly in each iteration as one may expected.

\subsection{Multi-scale}

Considering the above listed model sizes and calculation times it becomes evident that in order to model bigger surface samples while maintaining very short wavelength 
components of the surface roughness, several millions of DOFs may be necessary, making the memory requirements and the calculation times increase considerably.

A different approach to this problem would be the implementation of a multi-scale model. The interaction between the asperities in longer wavelengths could be taken into account in a coarse mesh while the asperities in contact could be calculated individually with a finer mesh that takes shorter wavelengths into account. The here presented model could be utilized as a fundamental building element for the implementation of such a multi-scale reduced model, similar to the one presented in 21.

\section{Conclusions and future work}

In this paper the theoretical foundation and some implementation details of a finiteelement model for the frictionless contact between nominally flat rough surfaces were presented. A series of calculation examples have shown that a model based on publicly available numerical libraries can achieve decent performance and provide sensible and useful results.

The first part of the calculated examples aimed to compare the model with the Greenwood \& Williamson theory. The results demonstrated a relatively good agreement with the theory. The real area of contact increases approximately proportional with the applied load while the mean contact pressure increases only slightly.

In the second part of the calculated examples, real surface topographies and an elastoplastic material behaviour were considered. The corresponding results seem to be coherent. The extent of plastic deformation is higher for less conformal contacting surfaces. Moreover, the model predicted correctly that loads higher than the ones applied during the operation of the real surfaces are necessary in order to cause further plastic deformation to the measured after the operation surfaces. It was also shown that for the specific materials pair studied in these examples, neglecting plasticity would have a significant influence on the calculated area of contact and mean contact pressure.

As a future work, the presented model is intended to be extended by including friction in form of shear stresses between the contacting surfaces and by implementing a multiscale calculation scheme. A further interesting application of the presented model would be to study the changes in the asperity heights distribution on unloaded surfaces that contain plastically deformed asperities.

\section{References}

[1] F. P. Bowden, D. Tabor, The area of contact between stationary and between moving surfaces, Proceedings of the Royal Society of London. Series A. Mathematical and Physical Sciences 169 (1939) 391-413.

[2] J. F. Archard, Elastic deformation and the laws of friction, Proceedings of the Royal Society of London. Series A. Mathematical and Physical Sciences 243 (1957) 190-205.

[3] J. A. Greenwood, J. B. P. Williamson, Contact of nominally flat surfaces, Proceedings of the Royal Society 295 (1966) 300-319.

[4] M. Ciavarella, J. Greenwood, M. Paggi, Inclusion of "interaction" in the greenwood and williamson contact theory, Wear 265 (2008) 729-734.

[5] R. L. Jackson, I. Green, A statistical model of elasto-plastic asperity contact between rough surfaces, Tribology International 39 (2006) 906-914.

[6] Majumdar, Bhushan, Fractal model of elastic-plastic contact between rough surfaces, Transactions of the ASME. Journal of Tribology 113 (1991) 1-11. 
[7] B. N. J. Persson, F. Bucher, B. Chiaia, Elastic contact between randomly rough surfaces: Comparison of theory with numerical results, PHYSICAL REVIEW -SERIES B- 65 (2002) 184106.

[8] Y. Ju, L. Zheng, A full numerical solution for the elastic contact of three-dimensional real rough surfaces, Wear 157 (1992) 151-161.

[9] Stanley, Kato, An fft-based method for rough surface contact, Transactions of the ASME. Journal of Tribology 119 (1997) 481-485.

[10] Hyun, Pei, Molinari, Robbins, Finite-element analysis of contact between elastic self-affine surfaces, Physical Review E (Statistical, Nonlinear, and Soft Matter Physics) 70 (2004) 26117-1-12.

[11] L. Pei, S. Hyun, J. Molinari, M. O. Robbins, Finite element modeling of elasto-plastic contact between rough surfaces, Journal of the Mechanics and Physics of Solids 53 (2005) 2385-2409.

[12] P. Wriggers, Computational Contact Mechanics, Springer, 2006.

[13] P. Alart, A. Curnier, A mixed formulation for frictional contact problems prone to newton like solution methods, Computer Methods in Applied Mechanics and Engineering 92 (1991) 353-375.

[14] Y. Renard, Generalized newton's methods for the approximation and resolution of frictional contact problems in elasticity, Computer Methods in Applied Mechanics and Engineering 256 (2013) 38 55.

[15] B. Herry, L. Di Valentin, A. Combescure, An approach to the connection between subdomains with non-matching meshes for transient mechanical analysis, International Journal for Numerical Methods in Engineering 55 (2002) 973-1003.

[16] A. Anandarajah, Computational methods in elasticity and plasticity : solids and porous media, Springer, 2010

[17] P. R. Amestoy, I. S. Duff, J. Koster, J.-Y. L'Excellent, A fully asynchronous multifrontal solver using distributed dynamic scheduling, SIAM Journal on Matrix Analysis and Applications 23 (2001) $15-41$.

[18] P. R. Amestoy, A. Guermouche, J.-Y. L'Excellent, S. Pralet, Hybrid scheduling for the parallel solution of linear systems, Parallel Computing 32 (2006) 136-156.

[19] K. Poulios, G. Svendsen, J. Hiller, P. Klit, Coefficient of friction measurements for thermoplastics and fibre composites under low sliding velocity and high pressure, Tribology Letters (2013).

[20] Y. Kadin, Y. Kligerman, I. Etsion, Unloading an elastic-plastic contact of rough surfaces, Journal of the Mechanics and Physics of Solids 54 (2006) 2652 - 2674.

[21] V. A. Yastrebov, J. Durand, H. Proudhon, G. Cailletaud, Rough surface contact analysis by means of the finite element method and of a new reduced model, Comptes Rendus Mecanique 339 (2011) 473. 